\title{
Therapeutic Challeng es of COVID-19 in a Patient Admitted to the Psychosomatic Ward: A Case Report
}

\author{
Razieh Salehian (i) ${ }^{1, *}$ and Mehdi Nasr Esfahani (iD ${ }^{2}$ \\ ${ }^{1}$ Department of Psychosomatic, Rasoul-e Akram Hospital, Iran University of medical sciences (IUMS), Tehran, Iran \\ ${ }^{2}$ Mental Health Research Center, School of Behavioral Sciences and Mental Health (Tehran Psychiatry Institute), Iran University of Medical Sciences (IUMS), Tehran, Iran \\ "Corresponding author: Department of Psychosomatic, Rasoul-e Akram Hospital, Iran University of medical sciences (IUMS), Tehran, Iran. Email: salehian.r@iums.ac.ir
}

Received 2020 November 27; Revised 2021 January 18; Accepted 2021 January 30.

\begin{abstract}
Introduction: Early diagnosis of 2019-nCoV infection is of great importance and can be challenging in psychiatric patients, especially when a mental illness such as somatoform disorder causes one or more bodily symptoms because the clinical features of this group of patients may be more confusing compared with non-psychiatric patients. On the other hand, treating this infection in psychiatric patients faces some challenges.

Case Presentation: A case of the 2019-nCoV infection is reported in a patient who was admitted to the psychosomatic ward with a diagnosis of somatic symptom disorder. The patient had a history of numerous unexplained physical complaints, usually complained of some new physical symptoms when informed of the time of his discharge. Although the possibility of misdiagnosis was high, based on examinations and some paraclinical evaluations, the patient underwent a simultaneous diagnosis of COVID-19. Unlike usual, he did not complain of any new physical complaints after informing of the 2019-nCoV infection and was willing to be discharged. Some diagnostic and therapeutic challenges regarding 2019-nCoV infection in the patient were examined.

Conclusions: There are three clinically relevant learning points to be noted from this case report. Firstly, the importance of paying attention to the patient's complaints in any mental patient, even disorders related to unexplained physical complaints. Secondly, the introduction highlights the differences in the care of patients with COVID-19 between psychiatric and non-psychiatric patients and the need for a multidisciplinary approach. Third, this introduction identifies a crucial diagnostic role for CT thorax in symptomatic patients with suspected COVID-19 because the false-negative rate with RT-PCR COVID-19 nasopharyngeal swabs is high.
\end{abstract}

Keywords: COVID-19, Psychosomatic, Somatic Symptom Disorder

\section{Introduction}

According to the fifth edition of the American Psychiatric Association's Diagnostic and Statistical Manual of Mental Disorders (DSM-5), somatic symptom disorder is diagnosed when one or more persistent somatic symptoms which are associated with excessive thoughts, feelings, and behaviors related to those symptoms were present $(1,2)$. Physicians face various challenges in diagnosing and treating these patients and find it difficult to treat this group of patients $(2,3)$. The introduction of this case explains the diagnostic and therapeutic challenges of 2019-nCoV infection (COVID-19) in a patient admitted to the hospital with a diagnosis of somatic symptoms disorder.

\section{Case Presentation}

A 31-year-old single man was admitted to the psychosomatic ward due to suicidal thoughts, self-cutting, and outbursts of anger after arguing with family members. He complained of severe insomnia and some physical symptoms, including severe headache, burning of the head, and dizziness despite adherence to the medication (sertraline $100 \mathrm{mg}$ per day and olanzapine $10 \mathrm{mg}$ at bedtime). Psychiatric interviews revealed that since the last 2 years following an emotional stressor, he has suffered from abdominal pain, hurt burn, nausea, and body pain, which has led to his frequent hospitalizations on the internal medicine and psychosomatic floor. Along with these symptoms, he frequently suffered from depressed mood, insomnia, loss of appetite, hopelessness, irritability, the thought of death, and interpersonal dysfunction over the past two years. At times, although the patient became asymptomatic e.g., gastrointestinal symptoms were relieved some days when his mood was euthymic, gastrointestinal symptoms quickly appeared upon experiencing new stressors or recalling previous stress. The patient has never had a history of physical aggression, no history of 
substance abuse, and the exam of his urine test for substance was negative. He left the job nine months ago due to severe gastrointestinal symptoms. Medical examinations and paraclinical assessments, including endoscopy, colonoscopy, and laboratory tests, were performed, but all were reported as normal. Brain CT scans and electroencephalogram did not show abnormal findings, and no evidence of memory impairment was found through clinical cognitive assessments. The patient did not have evidence for the episode of mania or psychosis and based on a dual diagnosis of the somatic symptom disorders and adjustment disorder with depressed mood, sertraline $100 \mathrm{mg}$ daily, olanzapine $5 \mathrm{mg}$ three times daily, gabapentin 300 $\mathrm{mg}$ twice a day, and clonazepam $1 \mathrm{mg}$ at bedtime were administrated.

The day after his admission, he complained of acute abdominal and chest pain. Chest X-rays, electrocardiogram, abdominal ultrasound, and laboratory examination, including myocardial enzymes, were examined. The results of paraclinical examinations were normal, except for liver enzymes that had doubled; levels of alanine aminotransferase, aspartate aminotransferase, and alkaline phosphatase were 129, 96, and 268 IU per liter, respectively; so the doses of sertraline, gabapentin, olanzapine, and clonazepam were reduced. Repetition of liver enzymes after seven days showed a decrease. He received several sessions of supportive psychotherapy because he did not have the skills to deal with acute stressors. Despite the reduction in drug dose, a significant improvement was observed in depressive symptoms, suicidal ideation, and physical symptoms.

The day that discharge planning was announced to the patient, he complained of a sore throat for the last two nights, without any other symptoms of COVID-19-like respiratory distress, shortness of breath or chest pain, cough, rhinorrhea, fever, muscle pain, and so on. Physical Examination showed erythema and some exudate in the throat, but the lungs were clear and vital signs were within normal ranges. Amoxicillin $500 \mathrm{mg}$ every 8 hours was administered, and due to the concomitant pandemic of COVID-19, some laboratory evaluations were requested as an emergency. To avoid the potential risk of transmitting infectious disease, the patient was transferred to an isolated room, and emergent infectious consultation was requested.

Pulse oximetry showed $\mathrm{PO}_{2}$ saturation as $96 \%$. The patient's new laboratory findings suggested an infection: $\mathrm{WBC}=9,900 / \mathrm{mm}^{3}, \mathrm{~L}=18 \%, \mathrm{Hb}=16.6 \mathrm{~g} / \mathrm{dL}, \mathrm{PLT}=289,000$ $/ \mathrm{mm}^{3}$ and $\mathrm{CRP}=26 \mathrm{mg} / \mathrm{L}$. The lung CT scan showed patchy multifocal multilobed pulmonary involvement in favor of COVID pulmonary involvement. The infectious disease specialist made a diagnosis of $2019-\mathrm{nCoV}$ infection for the patient and recommended real-time polymerasechain-reaction (RT-PCR) COVID-19 testing and initiation of treatment. The next day patient was discharged from the hospital, but some interventions were performed before discharge. He received a levofloxacin injection. Also, azithromycin $500 \mathrm{mg}$ daily, hydroxychloroquine $200 \mathrm{mg}$ every 12 hours was prescribed to use following discharge. Corrected QT Interval (QTC) was within the normal range, but considering the possibility of the emergence of respiratory distress and some drug interactions, the dose of clonazepam, gabapentin, and olanzapine was reduced before discharge. A training session was held to learn the principles of quarantine, symptoms indicating the severity of the disease, and the process of follow-up treatment. A nasopharyngeal swab was taken before discharge, although a negative result for 2019 -nCoV by RT-PCR assay was obtained two days after discharge. Because it takes about 48 hours to find the result for 2019-nCoV by RT-PCR assay, and in many cases, this test has false-negative results, many physicians diagnose 2019-nCoV infection in Iran based on the patient's symptoms and the results of non-specific tests such as CBC, CRP, especially evidence of the lung involvement with Coronavirus on CT scans.

\section{Discussion}

Psychiatric disorders, especially somatic symptom disorder, can interfere with the care of patients infected with the 2019-nCoV. These patients may report unexplained respiratory and other symptoms of COVID-19 after being informed of their infection, or conversely, may focus on other physical or mental symptoms and do not pay adequate attention to more important symptoms. On the other hand, the patients may experience an exacerbation of the primary disorder in the face of new stress, especially patients who did not have adequate defense mechanisms and intellectual function. Another issue that should be considered in mentally ill patients is that the patient may not have sufficient insight into the importance of using protective materials and social distance, leading to the transmission of the disease to others.

Psychiatric drugs have different physical side effects that should be considered in patients with COVID-19. Selective serotonin reuptake inhibitors (SSRIs) like sertraline can cause slowing of heart rate, sinus bradycardia, or sinus arrest $(4,5)$. Some antipsychotics are associated with QT interval prolongation, and if they are taken with other drugs such as azithromycin or chloroquine, which have the same risk, the risk of QT prolongation is further increased and can lead to be fatal ventricular arrhythmia (68). Benzodiazepines can cause respiratory depression, so 
short-acting benzodiazepines with the lowest dose are recommended if necessary $(6,9)$. Given that most psychiatric drugs have hepatic metabolism and they may inhibit the hepatic metabolism of other medications, it is essential to pay attention to the drug interaction of psychiatric medication with some of the drugs that commonly are used in COVID-19 $(6,10)$. In this patient, due to the recent increase in liver enzymes, there was a need to pay more attention to this issue, and liver enzyme tests were repeated.

One important issue is the psychological effects that an infected patient has on other patients. If other patients inform that a patient who was hospitalized at the same time is infected with coronavirus, they may become worried and anxious. Stigma is an important issue in both psychiatric disorders and infectious diseases. Stigma can lead to a social distance of the infected person from others. This type of behavior change can prevent the disease from spreading (11). However, stigma can prevent healthy behaviors and lead to disease severity, disease transmission, and problems in controlling the spread of infectious diseases (12). Although the patient always tried to attract the attention of others and play the role of the patient by expressing physical complaints, he was upset about his infection with COVID and did not want other patients to know about his infection.

Another issue that may be helpful in the recent pandemic is the crucial diagnostic role for thoracic CT scan in symptomatic patients who are suspected of COVID-19 since the false-negative rate with RT-PCR COVID-19 nasopharyngeal swabs is as high as $25 \%-30 \%$ (13).

\section{Footnotes}

Authors' Contribution: Study concept and design: RS and MNE. Drafting of the manuscript: RS and MNE. Critical revision of the manuscript for important intellectual content: RS and MNE.

Conflict of Interests: The authors have no conflict of interests.

Funding/Support: This case report has been registered in the research system (Pajoheshyar) of Iran University of Medical Sciences.

Informed Consent: Informed consent was obtained from the patient.

\section{References}

1. Gerstenblith TA, Kontos N. Somatic symptom disorders. In: Stern TA, Fava M, Wilens TE, Rosenbaum JF, editors. Massachusetts General Hospital comprehensive clinical psychiatry. 2nd ed. London: Elsevier; 2016. p. 348-57.

2. Dimsdale JE, Creed F, Escobar J, Sharpe M, Wulsin L, Barsky A, et al. Somatic symptom disorder: an important change in DSM. J Psychosom Res. 2013;75(3):223-8. doi: 10.1016/j.jpsychores.2013.06.033. [PubMed: 23972410].

3. Henningsen P. Management of somatic symptom disorder. Dialogues Clin Neurosci.2018;20(1):23-31. [PubMed: 29946208]. [PubMed Central: PMC6016049].

4. Shapiro PA, Critchfield AR. Cardiovascular Disorders. In: Sadock BJ, Sadock VA, Ruiz P, editors. Kaplan and Sadock's comprehensive textbook of psychiatry. 1. 10th ed. Philadelphia: Wolters Kluwer; 2017. p. 2186200.

5. Ungvari Z, Tarantini S, Yabluchanskiy A, Csiszar A. Potential Adverse Cardiovascular Effects of Treatment With Fluoxetine and Other Selective Serotonin Reuptake Inhibitors (SSRIs) in Patients With Geriatric Depression: Implications for Atherogenesis and Cerebromicrovascular Dysregulation. Front Genet. 2019;10:898. doi: 10.3389/fgene.2019.00898. [PubMed: 31616477]. [PubMed Central: PMC6764114].

6. Owen J, Crouse E, Kirkwood C, Levenson J. Psychopharmacology. In: Levenson JL, editor. The American Psychiatric Association Publishing Textbook of Psychosomatic Medicine and Consultation-Liaison Psychiatry.3rd ed. Washington, D.C: American Psychiatric Association Publishing; 2018. p. 1474-572. doi:10.1176/appi.books.9781615371990.jl36.

7. Beach SR, Celano CM, Sugrue AM, Adams C, Ackerman MJ, Noseworthy PA, et al. QT Prolongation, Torsades de Pointes, and Psychotropic Medications: A 5-Year Update. Psychosomatics. 2018;59(2):105-22. doi: 10.1016/j.psym.2017.10.009. [PubMed: 29275963].

8. Lambiase PD, de Bono JP, Schilling RJ, Lowe M, Turley A, Slade A, et al. British Heart Rhythm Society Clinical Practice Guidelines on the Management of Patients Developing QT Prolongation on Antipsychotic Medication. Arrhythm Electrophysiol Rev. 2019;8(3):161-5. doi: 10.15420/aer.2019.8.3.G1. [PubMed: 31463053]. [PubMed Central: PMC6702465].

9. Griffin C3, Kaye AM, Bueno FR, Kaye AD. Benzodiazepine pharmacology and central nervous system-mediated effects. Ochsner J. 2013;13(2):214-23. [PubMed: 23789008]. [PubMed Central: PMC3684331].

10. Telles-Correia D, Barbosa A, Cortez-Pinto H, Campos C, Rocha NB, Machado S. Psychotropic drugs and liver disease: A critical review of pharmacokinetics and liver toxicity. World J Gastrointest Pharmacol Ther. 2017;8(1):26-38. doi: 10.4292/wjgpt.v8.i1.26. [PubMed: 28217372]. [PubMed Central: PMC5292604].

11. Oaten M, Stevenson RJ, Case TI. Disease avoidance as a functional basis for stigmatization. Philos Trans $R$ Soc Lond B Biol Sci. 2011;366(1583):3433-52. doi: 10.1098/rstb.2011.0095. [PubMed: 22042920]. [PubMed Central: PMC3189356].

12. Deacon $\mathrm{H}$. Towards a sustainable theory of health-related stigma: lessons from the HIV/AIDS literature. Journal of Community \& Applied Social Psychology. 2006;16(6):418-25. doi:10.1002/casp.900.

13. Vepa A, Saleem A, Dharmaraj D, Afzaal Q. COVID-19, Diagnostic Difficulties and Acute Psychosis. Br J Med Pract. 2020;13(1). 